\title{
Niveles de homocisteína y polimorfismos de los genes de la MTHFR y la CBS en pacientes colombianos con trombosis venosa superficial y profunda
}

\author{
Claudia Ayala1, Reggie García1 ${ }^{1}$ Edith Cruz², Karol Prieto¹, Marta Bermúdez ${ }^{1,2}$ \\ 1 Instituto de Genética Humana, Pontificia Universidad Javeriana, Bogotá, D.C., Colombia \\ 2 Universidad Colegio Mayor de Cundinamarca, Bogotá, D.C., Colombia
}

Introducción. Se produce trombosis cuando en el sistema hemostático se desequilibran los mecanismos procoagulantes, anticoagulantes y fibrinolíticos, y se forman coágulos dentro de los vasos sanguíneos. Los factores de riesgo para el desarrollo de esta enfermedad pueden ser adquiridos o genéticos, polimorfismos o mutaciones en genes que conducen a hiperhomocisteinemia o que están comprometidos en las vías de coagulación.

Objetivos. Analizar, en una población colombiana con diagnóstico de trombosis venosa el perfil lipídico, los niveles de glucosa y homocisteína, y calcular las frecuencias alélicas y genotípicas de los polimorfismos c.677C>T del gen de la metilen-tetra-hidrofolato reductasa (MTHFR) y c. 699C>T, c. 1080 C>T, c.844ins68 del gen de la cistationina betasintasa (CBS).

Materiales y métodos. Se estudiaron 33 pacientes con sus respectivos controles. Las pruebas bioquímicas se realizaron por métodos colorimétricos y de inmunoensayo. Se utilizó la técnica de fragmentos de longitud polimórfica para la identificación de los polimorfismos mencionados. El estudio de asociación se hizo mediante la prueba de de ji al cuadrado.

Resultados. Se confirmó el papel de algunos factores de riesgo ya establecidos para el desarrollo de enfermedad trombótica venosa y se encontró un efecto protector del polimorfismo CBS c.699C>T para el riesgo de hipercolesterolemia con diferencia estadísticamente significativa en el grupo de los casos al compararlo con los controles. Por otra parte, se encontró una tendencia estadística que podría indicar un efecto protector del polimorfismo 844ins68 para el desarrollo de enfermedad trombótica venosa.

Conclusiones. No se encontraron diferencias estadísticamente significativas en los niveles de homocisteína entre el grupo de casos y de controles. Sin embargo, la variabilidad en las concentraciones plasmáticas fue mayor en los casos.

Palabras clave: homocisteína, polimorfismo genético, hiperhomocisteinemia, trombosis, aterosclerosis, cistationina betasintasa.

Homocysteine levels and polymorphisms of MTHFR and CBS genes in Colombian patients with superficial and deep venous thrombosis

Introduction. Thrombosis develops when the hemostatic system is incorrectly activated due to the unbalance between procoagulant, anticoagulant and fibrinolytic mechanisms allowing the formation of a clot within a blood vessel. The risk factors of this pathology can be acquired or can be genetic.

Objectives. To analyze in a Colombian population with diagnosis of venous thrombosis, lipid profile, glucose and homocystein levels, to calculate the alleles and genotypic frequencies of polymorphisms c.699 C>T, c.1080 C>T, c.844ins68 of the cystathionine B synthase and the c.677 C>T of the methylenetetrahydrofolate reductase (MTHFR) genes.

Materials and methods. Thirty three patients and their controls were studied. The biochemical test was carried out by colorimetric methods and immunoassay. In this survey we used the restriction fragments longitude polymorphism (RLFP) technique to identify the polymorphisms mentioned. The association study was performed through the chi square test.

Results. We confirmed that gene alterations increase risk for pathology; we found statistically significant differences in the group with hypercholesterolemia in presence of the polymorphism c.699 C>T in the $C B S$ gene, showing a protective effect in the individuals carrying this genetic variation. Likewise, we found a statistical trend for an eventual protective effect of the CBS c.844ins68 polymorphism to venous thrombotic disease.

Conclusions. There were not any statistically significant differences in homocystein levels between cases and controls; nevertheless, the variability in the plasma concentrations was greater in the group of cases.

Key words: homocysteine; polymorphism, genetic; hyperhomocysteinemia, thrombosis, atherosclerosis, cystathionine beta-synthase. 
La aterotrombosis es un proceso complejo que cursa de manera crónica y que se inicia en las paredes internas de arterias o venas. Los mecanismos que la producen son varios y su fisiopatología se ha explicado con lo que se conoce como la tríada de Virchow, la cual consiste en estasis sanguínea, daño endotelial y aumento en la capacidad de coagulación, factores que en conjunto o por separado pueden llevar al desarrollo de esta enfermedad (1). In vivo existe un equilibrio entre la formación de fibrina y la fibrinólisis; por lo tanto, la reducción del flujo sanguíneo, los cambios en la pared de los vasos y los cambios en la composición de la sangre ("hipercoagulabilidad"), pueden resultar en un desequilibrio que favorece la formación de fibrina que, finalmente, lleva a la formación del trombo oclusivo $(2,3)$. La presencia de un trombo en el sistema venoso, acompañado por una respuesta inflamatoria, se conoce como trombosis venosa, o tromboflebitis.

Esta enfermedad se presenta con una incidencia anual de 1 a 3 por cada 1.000 personas y afecta tanto a jóvenes como a ancianos. Las trombosis venosas que se producen en el sistema venoso profundo son más importantes que las del sistema venoso superficial, debido a la gravedad de sus potenciales complicaciones (embolismo pulmonar, síndrome postrombótico) (4-6).

Los factores de riesgo para el desarrollo de la trombosis venosa, pueden ser adquiridos o genéticos. Entre los factores de riesgo adquiridos, se encuentran la diabetes mellitus, las hiperlipidemias, el tabaquismo y el uso de anticonceptivos; en menor proporción, aparecen aquellos asociados con inmovilización, cirugía, trauma y embarazo.

Por otra parte, entre los factores de riesgo genéticos, descritos como asociados a la trombosis, se encuentran las anormalidades del sistema de la coagulación, tales como el factor $\mathrm{V}$ de Leiden, el déficit de antitrombina III, las proteínas C, la proteína $S$ y altos niveles de los factores procoagulantes (factor II, VIII, IX y XI) (2-6).

Recientemente, se ha descrito otro factor de riesgo genético involucrado en el desarrollo de la

\section{Correspondencia:}

Marta Bermúdez, Instituto de Genética Humana, Facultad de Medicina, Pontificia Universidad Javeriana, Carrera $7^{a}$ № 40- $^{-}$ 62, Bogotá, D.C., Colombia.

Teléfono: (+571) 320 8320, extensión 2791; fax: (571) 3208320 , extensión 2793.

martha.bermudez@javeriana.edu.co

Recibido: 15/07/09; aceptado:01/03/10 trombosis, conocido como hiperhomocisteinemia, alteración metabólica caracterizada por el aumento de los niveles de homocisteína en sangre (7). La homocisteína es un aminoácido sulfurado que no es incorporado en las proteínas y, por ende, no es un constituyente de la dieta. Este aminoácido se forma exclusivamente como un producto intermediario del metabolismo de la metionina. Varias enzimas intervienen en la vía metabólica de la metioninahomocisteína, entre ellas, la metilentetrahidrofolato reductasa (MTHFR) y la cistationina betasintasa (CBS) $(7,8)$.

Según Refsum et al. (9), si los niveles de homocisteína total en plasma están en el rango entre 14 y $100 \mu \mathrm{mol} / \mathrm{L}$, se considera como hiperhomocisteinemia moderada, y si los niveles de homocisteína son mayores de $100 \mu \mathrm{mol} / \mathrm{L}$, se clasifica como hiperhomocisteinemia grave u homocistinuria. Bermúdez et al., en 2006 (10), describieron los niveles de homocisteína basal en población adulta y sana de Colombia. El valor promedio encontrado fue de $10,52 \mu \mathrm{mol} / \mathrm{L}$ con una desviación estándar de 2,86 $\mu \mathrm{mol} / \mathrm{L}$.

Existen varios factores que influyen en los niveles plasmáticos de homocisteína, como la edad, el sexo, el tabaquismo, la nutrición, ciertos medicamentos y alteraciones genéticas como consecuencia de mutaciones o polimorfismos en los genes de alguna de las enzimas involucradas en el metabolismo de la metionina-homocisteína.

Las vitaminas $B_{6,} B_{12} y$ el ácido fólico intervienen en el funcionamiento de las enzimas MTHFR y CBS. La deficiencia nutricional de alguna de estas vitaminas conduce a la elevación de los niveles intracelulares de homocisteína y, como consecuencia, a hiperhomocisteinemia.

Por otro lado, los fármacos como el metotrexato y la fenitoína, que intervienen en el metabolismo del folato, y otros medicamentos, como las ciclosporinas, los antidiabéticos (metformina), los hipolipidémicos, la terapia de reemplazo hormonal y los anticonceptivos orales, también influyen en los niveles de la homocisteína plasmática $(11,12)$.

Con relación a los factores genéticos que se asocian a hiperhomocisteinemia, está el gen de la MTHFR, que codifica la enzima metilentetrahidrofolato reductasa. La presencia de cambios en este gen puede llevar a que el metabolismo de la metioninahomocisteína no sea regulado de forma adecuada.

El gen de la MTHFR está localizado en el cromosoma 1 p36.3 y el ADNc tiene 2,2 kb y 11 
exones; entre los polimorfismos descritos está la transición c.677C > T, que resulta en una variante termolábil de la enzima, debido a que la enzima codificada por este alelo tiene una actividad reducida a $37^{\circ} \mathrm{C}$. Este cambio crea un sitio de restricción y la sustitución de un residuo de alanina por valina en la proteína $(11,13,14)$.

Por otro lado, el gen de la enzima CBS que codifica la enzima cistationina $\beta$ sintasa, tiene 28.046 pares de bases y 23 exones, y se encuentra localizado en la región subtelomérica de la banda 21q22.

Con el ADN genómico aislado a partir de sangre periférica y con iniciadores apropiados, se han identificado los diferentes polimorfismos genéticos de la MTHFR y de la CBS, que están asociados a la enfermedad aterotrombótica $(13,14)$. Entre éstos, están el c.699 C>T, el c.1080 C>T y el c.844ins68 del gen de la CBS.

Algunos autores han asociado la enfermedad aterotrombótica con los polimorfismos c.C677T de la MTHFR y c.C699T, C1080T y c.844ins68 de la CBS. Sin embargo, otros autores no han encontrado esta relación (15). Gemmati et al., en 1999 (16), en un estudio de casos y controles en los que incluyeron 160 casos con enfermedad 
de Bogotá (Cundinamarca) y del Hospital San Rafael del municipio de Pacho (Cundinamarca), con muestreo por conveniencia.

Se incluyeron los pacientes que cumplían con el siguiente criterio: diagnóstico confirmado de trombosis venosa por ecografía Doppler dúplex realizada durante el evento. Se excluyeron del estudio los individuos que no quisieron participar del mismo y quienes tenían una enfermedad de base que generara un estado protrombótico (síndrome antifosfolípido, lupus eritematoso sistémico, coagulopatía de origen genético o cáncer).

\section{Aspectos éticos}

A todo paciente que ingresó al estudio se le dieron a conocer los procedimientos por realizar y se les informó que esta investigación era considerada de riesgo mínimo; luego de la firma del consentimiento informado, se procedió a aplicar el cuestionario sobre antecedentes clínicos.

\section{Estudio bioquímico}

Las pruebas bioquímicas se realizaron por métodos colorimétricos y de inmunoensayo de polarización fluorescente (Fluorescence Polarization Immunoassay, FPIA).

\section{Estudio molecular}

Análisis de polimorfismos: el ADN del genoma se aisló de sangre periférica mediante el método de precipitación salina. Para la identificación del polimorfismo c.677C/T del gen de la MTHFR, se amplificó el exón 1 y se utilizaron los iniciadores forward: 5'TGAAGGAGAAGGTGTCTGCGGGA3', y reverse: 5' AGGACGGTGCGGTGAGTG3', con las condiciones experimentales de la PCR descritas por Bermúdez et al. (10). El análisis de restricción se realizó con la enzima Taq I. La presencia del polimorfismo crea sitio de restricción y se generaron los fragmentos 177 y 21 , pares de bases que se separaron en gel de poliacrilamida (10).

Polimorfismo c.699C/T del gen de la CBS: se amplificó el exón 6 con los iniciadores forward 5' CAGCAACCCCCTGGCTCAGT3' y reverse 5' CAGCCATGCCCTGTGTTTGCTATT3', con condiciones experimentales de PCR descritas por Bermúdez et al. (10). Se obtuvo un fragmento de 289 pares de bases, al digerir con la enzima de restricción Rsal y separar los productos en gel de poliacrilamida, se evidenciaron fragmentos de 177,92 y 20 pares de bases, si el genotipo era C/C y, de 177 y 112 pares de bases, si el genotipo eran T/T (10).
Polimorfismo c.1080 C>T del gen de la CBS: se amplificó el exón 10 con los primers forward 5' CAGTGCCCACCCCAGCTCATTA3' y reverse 5' GGCCTCCTCCCCTCCCAGTTCT3', con las condiciones experimentales de PCR descritas por Bermúdez et al. (10). Se obtuvo un fragmento de 465 pares de bases, al digerir con la enzima de restricción BstUl y separar los productos en gel de poliacrilamida, se observaron fragmentos de 219 , 178,69 y 20 pares de bases, si el genotipo era C/C; el polimorfismo $\mathrm{T}$, hace que se pierda un punto de corte con la enzima de restricción, produciendo los fragmentos 246 y 219 (10).

c.844 Inserción 68 pb del gen de la CBS: se utilizaron los iniciadores forward 5' CCGCAGG GTGGTCTGTCTGGACTG3' y reverse 5' AGCCC CACTCAGCATCCGTGTGAC3', con las siguientes condiciones de PCR: un ciclo de dos minutos a $94^{\circ} \mathrm{C}, 35$ ciclos con las condiciones de PCR descritas por Bermúdez et al. (10). Se obtuvo un fragmento de 805 pares de bases al separar los productos en agarosa al $2 \%$, en ausencia de la inserción de 68 pares de bases y de 873 pares de bases, cuando está presente la inserción (10).

Análisis estadístico: los resultados fueron almacenados y codificados en una base de datos en el programa Excel ${ }^{\circledR}$, Microsoft Office 2007. Los grupos de casos y controles fueron ajustados para sexo y edad ( \pm 2 años). Se realizó estadística descriptiva: promedios, desviaciones estándar, para las variables numéricas y porcentajes de aparición del evento para las variables categóricas. Asimismo, se analizó el riesgo para el desenlace atribuido a los factores de riesgo, mediante el cálculo de odds ratio (OR), prueba de ji al cuadrado y el planteamiento de pruebas de hipótesis con el fin de determinar si existía asociación entre las variables y la enfermedad o si no la había. Se tomó un nivel de confianza del 95\% para evaluar la significancia estadística. Las pruebas estadísticas se realizaron con los programas Epidat, versión 3.1, y Stata, versión 8.0.

\section{Resultados}

Se estudió una población de 33 personas con diagnóstico de trombosis venosa profunda y superficial, y 33 personas sanas, cada grupo conformado por 26 mujeres y 7 hombres ajustados por sexo y edad \pm 2 años (casos: 49,15 años $\pm 13,81$; controles: 47,52 años $\pm 12,98$ ), procedentes de Bogotá y del municipio de Pacho, Cundinamarca. 
Los individuos del grupo de estudio se evaluaron, en promedio, a los 39,52 meses $(D E=35,43$ ) después de presentar el primer evento. A partir de los datos de la encuesta realizada, se encontró que, del total de personas con enfermedad trombótica venosa, $24,24 \%$ presentaron trombosis venosa superficial, $66,66 \%$ trombosis venosa profunda y $21,21 \%$ tromboembolismo pulmonar asociado a la enfermedad trombótica venosa. En cuanto a la frecuencia del evento trombótico venoso, encontramos que $39,39 \%$ tuvieron un evento único, $27,27 \%$ sufrieron dos eventos, $9,09 \%$ presentaron tres o más eventos y en $24,24 \%$ de los casos no fue posible especificar la recurrencia del evento.

Entre los factores de riesgo conocidos evaluados por la encuesta, encontramos que $18,18 \%$ de los casos tenían antecedentes de tabaquismo, 9,09\% fumaban en el momento de ocurrencia del evento y $9,09 \%$ fumaban en el momento de la encuesta.

En cuanto a la valoración del índice de masa corporal, es importante mencionar que 6,06\% tenían bajo peso al momento de la evaluación, $42,42 \%$ estaban en el rango normal, $27,27 \%$ presentaron sobrepeso, $12,12 \%$ tenían obesidad tipo I, $12,12 \%$ tenían obesidad tipo II y ningún individuo fue clasificado como obesidad tipo III; finalmente, se obtuvo una media de 26,21 con desviación estándar de 5,81.

Otro aspecto importante evaluado fue el uso de hormonas femeninas, los cuales han sido asociados por diversos autores con el incremento de aparición de casos de trombosis venosa (1820). En nuestro estudio se encontró que $53,84 \%$ de las mujeres con enfermedad venosa trombótica usaron anticonceptivos orales e inyectables con componente estrogénico, antes de presentar el evento, $11,53 \%$ los utilizan en la actualidad, mientras que $15,38 \%$ estuvieron expuestas durante el evento.

Es de relevancia destacar las frecuencias encontradas para factores de riesgo modificables en personas que han sufrido eventos trombóticos, por lo que creemos que se debe reforzar y tener claridad en la educación e indicaciones al paciente en la consulta externa, tanto en medicina general como en medicina especializada, para disminuir el riesgo de presentar estas entidades. Además, es importante destacar que $7,69 \%$ de los casos de sexo femenino desarrollaron el episodio de trombosis durante el embarazo y $15,15 \%$ de los casos, durante un periodo posoperatorio.
En cuanto al análisis de las pruebas de laboratorio, se encontraron diferencias estadísticamente significativas en los valores promedio de colesterol total, triglicéridos y colesterol LDL, entre los grupos de casos y controles. Además, es importante resaltar que se encontraron diferencias estadísticamente significativas en la variabilidad de los valores de colesterol total, triglicéridos, colesterol LDL, glucosa y homocisteína, entre los casos y los controles (cuadro 1).

Al determinar el grado de riesgo de presentar trombosis venosa con hipercolesterolemia (>200 mg/dl), hipertrigliceridemia (>150 mg/dl), hipocolesterolemia HDL $(\leq 50 \mathrm{mg} / \mathrm{dl})$, hiperocolesterolemia LDL (>150 mg/dl) e hiperglucemia en ayunas (>110 mg/dl), no se observaron diferencias estadísticamente significativas que modificaran el riesgo de sufrir la enfermedad (cuadro 2). En cuanto a la evaluación de hiperhomocisteinemia $(>17 \mu \mathrm{mol} / \mathrm{L})$, se encontraron tres personas con hiperhomocisteinemia en el grupo de los casos, con valores de 19,3 $\mu \mathrm{mol} / \mathrm{L}, 23,6$ y $23,9 \mu \mathrm{mol} / \mathrm{L}$, mientras que en el grupo control no se encontró ninguna persona con aumento de la homocisteinemia.

Con respecto a la valoración del equilibrio de HardyWeinberg para los genotipos de los polimorfismos evaluados, se encontraron en desequilibrio los polimorfismos c.C677T del gen de la MTHFR y c.C1080T del gen de la CBS, en el grupo de casos. Para este análisis se utilizó el método de cadenas de Markov en el programa GENEPOP, versión 4.0 (cuadros 3 y 4 ).

No se encontraron asociaciones con diferencias estadísticamente significativas, al analizar la relación entre los diferentes factores de riesgo y los polimorfismos en los genes de la CBS y de la MTHFR en los grupos de casos y controles, excepto para el polimorfismo CBS c.C699T, que mostró un efecto protector para hipercolesterolemia en los pacientes con trombosis venosa $(\mathrm{OR}=0,030$; IC95\% 0,003-0,305; $p=0,0004$ ).

En cuanto a la evaluación de la asociación de los polimorfismos genéticos con trombosis venosa superficial y profunda, no se encontraron diferencias estadísticamente significativas entre los grupos de casos y controles, pero es importante resaltar la tendencia estadística del polimorfismo de la CBS 844 ins68 como factor protector para el desarrollo de esta enfermedad (OR=0,11; IC95\% 0,01-1,00; $p=0,0539$ ). 
Cuadro 1. Características del grupo de casos y de los controles.

\begin{tabular}{|c|c|c|c|c|c|c|}
\hline \multirow{2}{*}{$\begin{array}{l}\text { Variables } \\
\text { Colesterol total }\end{array}$} & \multicolumn{2}{|c|}{$\begin{array}{l}\text { Casos } \\
\bar{X} \pm D E\end{array}$} & \multicolumn{2}{|c|}{$\begin{array}{c}\text { Controles } \\
\bar{x} \pm D E\end{array}$} & \multirow{2}{*}{$\begin{array}{c}p 1 \\
0,0098\end{array}$} & \multirow{2}{*}{$\begin{array}{c}\text { p2 } \\
0,0001\end{array}$} \\
\hline & 212,48 & 59,95 & 181,19 & 29,41 & & \\
\hline Triglicéridos & 123,31 & 58,46 & 94,09 & 37,07 & 0,0187 & 0,0119 \\
\hline Colesterol HDL & 45,48 & 12,83 & 48,60 & 9,59 & 0,2674 & 0,1047 \\
\hline Colesterol LDL & 142,10 & 62,12 & 115,84 & 29,23 & 0,0331 & 0,0001 \\
\hline Glucosa & 95,86 & 27,56 & 90,58 & 16,59 & 0,3501 & 0,0052 \\
\hline Homocisteína & 9,87 & 4,71 & 9,88 & 2,96 & 0,9918 & 0,0104 \\
\hline
\end{tabular}

HDL: lipoproteína de alta densidad; LDL: lipoproteína de baja densidad; $\bar{x} \pm D E$ : media \pm desviación estándar; $p 1$ : comparación entre medias; $p 2$ : comparación de varianzas

${ }^{*} p<0,05$

Finalmente, al realizar el análisis multivariado no se encontraron diferencias estadísticamente significativas entre el grupo de casos y el grupo control para los factores de riesgo evaluados.

\section{Discusión}

En relación con la valoración de los factores de riesgo ya conocidos, los resultados de nuestro estudio parecen validar la importancia del componente metabólico en el desarrollo de la enfermedad trombótica venosa, ya que se observaron niveles más altos en las medias de las concentraciones de colesterol total, triglicéridos y colesterol LDL en las personas con la enfermedad, en comparación con el grupo control. Además, encontramos diferencias estadísticamente significativas en la variabilidad de las concentraciones de colesterol

Cuadro 2. Relación entre trombosis y factores de riesgo.

\begin{tabular}{lccc}
\hline Factor de riesgo & OR & IC 95\% & $\boldsymbol{p}$ \\
\hline Colesterol total & 2,92 & $0,94-9,02$ & 0,114 \\
Triglicéridos & 4,17 & $0,79-21,85$ & 0,1487 \\
Colesterol HDL & 1,44 & $0,54-3,84$ & 0,9138 \\
Colesterol LDL & 2,80 & $0,84-9,25$ & 0,1696 \\
Glucosa & 1,00 & $0,22-4,38$ & 221,00 \\
\hline
\end{tabular}

OR: odds ratio; IC95\%: intervalo de confianza del 95\% total, triglicéridos, colesterol LDL, glucemia basal y homocisteína plasmática en los casos; esto indica mayor homogeneidad en las concentraciones para estos metabolitos en el grupo control y posibles diferencias en el comportamiento biológico (cuadro 1). Sin embargo, al clasificar el nivel de cada metabolito como elevado o disminuido, de acuerdo con los valores umbrales establecidos por cada estuche diagnóstico, y evaluar el riesgo para la enfermedad trombótica venosa, no se encontraron diferencias estadísticamente significativas (cuadro 2).

En este estudio, con respecto a los niveles de homocisteína, no se encontraron diferencias estadísticamente significativas en la comparación de medias entre los grupos de casos y controles, aunque sí hubo diferencias estadísticamente significativasenlavariabilidaddelasconcentraciones plasmáticas, mayor en el grupo de los casos. Este hallazgo en la variación en el comportamiento del metabolismo de la metionina-homocisteína, hace sospechar un posible papel de esta vía metabólica en la enfermedad venosa trombótica. Además, cabe resaltar que se encontraron tres individuos con hiperhomocisteinemia que presentaban la enfermedad, mientras que no se encontró ninguno en el grupo control.

Cuadro 3. Frecuencias genotípicas y fenotípicas en el grupo de casos.

\begin{tabular}{|c|c|c|c|c|c|c|c|c|c|}
\hline \multirow[b]{2}{*}{ Polimorfismos } & \multicolumn{6}{|c|}{ Genotipos observados grupo de controles $(n=33)$} & \multirow[b]{2}{*}{$\begin{array}{l}\text { Frecuencia } \\
\text { del alelo } w^{a}\end{array}$} & \multirow[b]{2}{*}{$\begin{array}{l}\text { Frecuencia } \\
\text { del alelo } \mathrm{M}^{\mathrm{b}}\end{array}$} & \multirow[b]{2}{*}{ HW: $p$} \\
\hline & W/Wa & $\%$ & W/M & $\%$ & $M / M^{b}$ & $\%$ & & & \\
\hline MTHFR C677T & 2 & 6 & 22 & 67 & 9 & 27 & 0,39 & 0,61 & $0,0372^{*}$ \\
\hline CBS C699T & 19 & 58 & 13 & 39 & 1 & 3 & 0,77 & 0,23 & 1 \\
\hline CBS C1080T & 11 & 33 & 22 & 67 & 0 & 0 & 0,67 & 0,33 & $0,0051^{*}$ \\
\hline CBS 844ins68 & 32 & 97 & 1 & 3 & 0 & 0 & 0,98 & 0,03 & 1 \\
\hline
\end{tabular}

MTHFR: metilen-tetra-hidrofolato reductasa; CBS: cistationina betasintasa; $\mathrm{W}^{\mathrm{a}}$ : alelo normal; $\mathrm{M}^{\mathrm{b}}$ : alelo mutado ; *HW: equilibrio de Hardy Weinberg.

Valor de p con nivel de confianza del 95\% para equilibrio de Hardy Weinberg, $p<0,05$ indica desequilibrio de Hardy Weinberg. 
Cuadro 4. Frecuencias genotípicas y fenotípicas en el grupo control.

\begin{tabular}{|c|c|c|c|c|c|c|c|c|c|}
\hline \multirow[b]{2}{*}{ Polimorfismos } & \multicolumn{6}{|c|}{ Genotipos observados grupo de controles $(n=33)$} & \multirow{2}{*}{$\begin{array}{l}\text { Frecuencia } \\
\text { del alelo } w^{a}\end{array}$} & \multirow{2}{*}{$\begin{array}{l}\text { Frecuencia } \\
\text { del alelo } \mathbf{M}^{\mathrm{b}}\end{array}$} & \multirow[b]{2}{*}{ HW: $p$} \\
\hline & W/Wa & $\%$ & W/M & $\%$ & $\mathbf{M} / \mathbf{M}^{\mathrm{b}}$ & $\%$ & & & \\
\hline MTHFR C677T & 5 & 15 & 23 & 70 & 5 & 15 & 0,50 & 0,50 & 0,383 \\
\hline CBS C699T & 19 & 58 & 13 & 39 & 1 & 3 & 0,77 & 0,23 & 0,956 \\
\hline CBS C1080T & 18 & 55 & 12 & 36 & 3 & 9 & 0,73 & 0,27 & 0,973 \\
\hline CBS 844ins68 & 26 & 79 & 7 & 21 & 0 & 0 & 0,89 & 0,11 & 1 \\
\hline
\end{tabular}

MTHFR: metilen-tetra-hidrofolato reductasa; CBS: cistationina betasintasa; Wa: Alelo normal; $\mathrm{M}^{\mathrm{b}}$ : alelo mutado; HW: equilibrio de Hardy Weinberg

* Valor de $\mathrm{p}$ con nivel de confianza del 95\% para equilibrio de Hardy Weinberg, $p<0,05$ indica desequilibrio de Hardy Weinberg.

Aunque se han postulado diversos mecanismos para explicar el papel fisiopatológico de la homocisteína en varias enfermedades, aún no están completamente esclarecidos. Los hallazgos de la literatura sugieren que los niveles de homocisteína podrían estar relacionados con estrés oxidativo, biodisponibilidad del óxido nítrico, funcionamiento del retículo endoplásmico, proliferación de células del músculo liso, propiedades elásticas de la pared vascular, disfunción endotelial, activación plaquetaria y del sistema de coagulación, y de esta forma, genera un estado protrombótico.

También es posible que, por medio de mecanismos genéticos y epigenéticos, pueda alterar la expresión de genes asociados a enfermedades en las cuales la homocisteína ha sido involucrada (13,21-23).

Cabe aclarar que, aunque los polimorfismos MTHFR c.677 C>T y CBS c.1080 C>T no se encontraron en equilibrio de Hardy Weinberg en el grupo de casos, es probable que este hallazgo se deba al tamaño de muestra y no a su papel en el desarrollo de esta enfermedad.

Es importante señalar el efecto protector para hipercolesterolemia conferido por el polimorfismo CBS c. 699 C>T en los pacientes con trombosis venosa. Este hallazgo es relevante debido a la asociación reportada en la literatura entre los niveles de homocisteína y el metabolismo lipídico. Los niveles de homocisteína parecen intervenir en la síntesis de colesterol, funcionamiento, almacenamiento y transporte del retículo endoplásmico. Por consiguiente, se especula que los niveles elevados de homocisteína conllevan a una alteración en la regulación de la biosíntesis de colesterol y, por esta razón, se destaca la importancia de evaluar el papel de los factores genéticos (polimorfismo de la CBS como el c.699 C>T) y ambientales involucrados en la vía metabólica de la metionina-homocisteína en la regulación de las concentraciones plasmáticas de colesterol (23).

Aunque la mayoría de las mutaciones en el gen de la CBS son raras, se ha reportado que la inserción de 68pb en el exón 8 del gen de la CBS es común, encontrándose en estado heterocigoto, aproximadamente, en $12 \%$ de la población general $(24,25)$. Al evaluar la frecuencia de los polimorfismos genéticos analizados para trombosis venosa, no se encontraron diferencias estadísticamente significativas $y$, por ende, incremento en el riesgo, pero cabe destacar una tendencia estadística de un posible efecto protector del polimorfismo CBS 844 ins68 en el desarrollo de la enfermedad; se encontró una frecuencia (11\%) de este polimorfismo en estado heterocigoto similar a la reportada a nivel mundial en el grupo control y de $3 \%$ en los casos.

Se resalta, además, que no se encontraron individuos homocigotos para este polimorfismo ni en los casos ni en los controles. El caso del polimorfismo CBS 844ins68 es llamativo, debido a la diversidad de resultados encontrados en la literatura al evaluar su papel en el desarrollo de enfermedad trombóticavascularehiperhomocisteinemia $(24,25)$. Grossmann et al., en 2002 (26), encontraron que el polimorfismo CBS c.844ins68, al ser evaluado en pacientes con trombosis venosa profunda contra un grupo control sano, daba un OR de 0,45 (IC95\% 0,27-0,77), lo cual podría indicar un efecto protector de este polimorfismo en la enfermedad. Mientras, autores como Bonini-Domingos et al., en 2005 (27), reportaron que el polimorfismo CBS 844ins68 en estado heterocigoto es un factor de riesgo para trombosis venosa profunda en población brasilera, también, se encuentran reportes que indican que la combinación del polimorfismo CBS c.844ins68 con la variante termolábil MTHFR puede incrementar el riesgo de enfermedad oclusiva arterial y venosa (27-30). 
Por otro lado, también se encuentran reportes en la literatura que indican aumento en la actividad enzimática de la CBS y un efecto protector de este polimorfismo para hiperhomocisteinemia (26). Con respecto a este punto, nuestros resultados no evidencian diferencias estadísticamente significativas entre los individuos con enfermedad trombótica venosa y el grupo control (OR: 0,14; IC95\% 0,011,$24 ; p=0,1048$ ), en cuanto a la presencia de los polimorfismos CBS c.844ins68 y MTHFR c.677 $\mathrm{C}>\mathrm{T}$ en estado heterocigoto.

Con este estudio se realizó una aproximación a la evaluación del papel de la hiperhomocisteinemia y de polimorfismos en genes involucrados en la vía de la homocisteína-metionina en el desarrollo de enfermedades multifactoriales como la enfermedad trombótica venosa, en población colombiana. Sin embargo, es necesario incrementar el tamaño de la muestra para tener una mejor estimación de los parámetros y frecuencias en las variables categóricas.

Finalmente, consideramos que es muy importante continuar el estudio del papel de la homocisteína y los factores genéticos y ambientales que regulan la vía metabólica de la homocisteína-metionina en la enfermedad vascular trombótica, para identificar la población en riesgo, y establecer estrategias para la prevención y el manejo de estas enfermedades.

\section{Agradecimientos}

Al Instituto de Genética Humana de la Pontificia Universidad Javeriana, Bogotá (Colombia), por su financiamiento. A Fernando Suárez e Ignacio Zarante, por su colaboración en la resolución de preguntas surgidas en el análisis del estudio $y$, de manera especial, a los pacientes.

\section{Conflicto de interés}

Los autores declaramos que no existe un posible conflicto de intereses en este manuscrito.

\section{Financiación}

Este estudio fue financiado por el Instituto de Genética Humana de la Pontificia Universidad Javeriana, Bogotá, D.C.

\section{Referencias}

1. Solymoss S. Risk factors for thromboembolism: pathophysiology and detection. CMAJ. 2000;163:991-4.

2. Kamphuisen PW, Eikenboom CC, Bertina RM. Elevated factor VIII levels and the risk of thrombosis. Arterioscler Thromb Vasc Biol. 2001;21:731-8.

3. Rosendaal FR. Venous thrombosis: the role of genes environment and behavior. Hematology. 2005;1:1-12.
4. Pendás JA, Estébanez RV. Trombosis venosa. Guías Clínicas. 2002; 26:1-5.

5. Doggen CJ, Smith NL, Lemaitre RN, Heckebert SR, Rosendaal FR, Psaty BM. Serum lipid levels and the risk of venous trombosis. Arterioscler Thromb Vasc Biol. 2004;24:1970-5.

6. Aznar J, Vaya A, Estellés A, Mira Y, Segui R, Villa P, et al. Risk of venous trombosis in carriers of the prothrombin G20210A variant and factor $V$ Leiden and their interaction with oral contraceptives. Haematologica. 2000;85:1271-6.

7. Eldibany MM, Caprini JA. Hyperhomocysteinemia and thrombosis. Arch Pathol Lab Med. 2007;131:837-72.

8. Loscalzo J. Homocysteine-mediated thrombosis and angiostasis in vascular pathobiology. J Clin Invest. 2009;119:3203-5.

9. Refsum H, Ueland PM, Nygard O, Vollset SE. Homocysteine and cardiovascular disease. Annu Rev Med. 1998;49:31-62.

10. Bermúdez M, Briceño I, Gil F, Bernal J. Homocisteína y polimorfismos de la cistationina $\beta$ sintasa $y$ metilentetrahidrofolato-reductasa en población sana de Colombia. Colomb Med. 2006;37:46-52.

11. Fonseca V, Suba SC, Fink LM. Hyperhocysteinemia and the endocrine system: implications for atherosclerosis and trombosis. Endocr Rev. 1999;20:738-59.

12. Nadir Y, Hoffman R, Brenner B. Association of homocysteine, vitamin B12, folic acid, and MTHFR C677T in patients with a thrombotic event or recurrent fetal loss. Ann Hematol. 2007;86:35-40.

13. Alpert MA. Homocysteine, atherosclerosis and thrombosis. South Med J. 1999;92:858-64.

14. Varga EA, Sturm AC, Misita CP, Moll S. Homocysteine and MTHFR mutations relation to thrombosis and coronary artery disease. Circulation. 2005;111:289-93.

15. Spiroski I, Kedev S, Antov S, Arsov T, Krstevska M, Dzhekova-Stojkova $\mathrm{S}$, et al. Association of methylenetetrahydrofolate reductase (MTHFR-677 and MTHFR-1298) genetic polymorphisms with occlusive artery disease and deep venous thrombosis in Macedonians. Croat Med J. 2008;49:39-49.

16. Gemmati D, Serino ML, Trivellato C, Fiorini S, Scapoli GL. C677T substitution in the methylenetetrahydrofolate reductase gene as a risk factor for venous thrombosis and arterial disease in selected patients. Haematologica. 1999;84:824-8.

17. Bezemer ID, Doggen KJ, Vos HL, Rosendaal FR. No association between the common MTHFR 677C $\square \mathrm{T}$ polymorphism and venous thrombosis. Arch Intern Med. 2007;167:497-501.

18. Orhan AL, Okuyan E, Okcun B, Nurkalem Z. Plasma homocysteine level and left ventricular thrombus formation in acute anterior myocardial infarction patients following thrombolytic therapy with t-PA. Thromb Res. 2009;124:65-9.

19. Vandenbroucke JP, Rosing J, Bloemenkamp KW, Middeldorp S, Helmerhorst FM, Bouma BN, et al. Oral contraceptives and the risk of venous thrombosis. N Engl $\mathrm{J}$ Med. 2001;344:1537-5. 
20. Rosendaal FR, Helmerhorst FM, Vandenbroucke JP. Female hormones and thrombosis. Arterioscler Thromb Vasc Biol. 2002;22:201-10.

21. D’Angelo A, Mazzola G, Crippa L, Fermo I, D’Angelo SV. Hyperhomocysteinemia and venous thromboembolic disease. Haematologica. 1997;82:211-9.

22. Undas A, Brozek J, Jankowski M, Siudak Z, Szczeklik A, Jakubowski H. Plasma homocysteine affects fribrin clot permeability and resistance to lysis in human subjects. Arterioscler Thromb Vasc Biol. 2006;26:1397-04.

23. Sharma $P$, Senthilkumar RD, Brahmachari $V$, Sundaramoorthy E, Mahajan A, Sharma A, et al. Mining literature for a comprehensive pathway analysis: A case study for retrieval of homocysteine related genes for genetic and epigenetic studies. Lipids Health Dis. 2006;5:1-19.

24. Tsai MY, Bignell M, Yang F, Welge BG, Graham KJ, Hanson NQ. Polygenic influence on plasma homocysteine: association of two prevalent mutations, the 844ins68 of cystathionine b-synthase and A2756G of methionine synthase, with lowered plasma homocysteine levels. Atherosclerosis. 2000;149:131-7.

25. Lievers KJ, Kluijtmans LA, Blom HJ. Genetics of hyperhomocysteinaemia in cardiovascular disease. Ann Clin Biochem. 2003;40:46-59.
26. Grossman R, Schwender S, Geisen U, Schambeck C, Merati G, Walter U. CBS 844ins68, MTHFR TT677 and EPCR 4031ins23 genotypes in patients with deep-vein thrombosis. Thromb Res. 2002;107:13-5.

27. Bonini-Domingos CR, Zamaro PJ, Mendiburu CF, Sanchez FE, Cintra JR, de Godoy JM, et al. Frequency of the 844ins 68 mutation on the cystathionine $\beta$-synthetase gene in deep venous thrombosis patients. Rev Bras Hematol Hemoter. 2005;1:12-5.

28. Franchis R, Fermo I, Mazzola G, Sebastio G, Di Minno $\mathrm{G}$, Coppola A, et al. Contribution of the cystathionine b synthase gene (844ins68) polymorphism to the risk of earlyonsent venous and arterial occlusive disease and of fasting hyperhomocysteinemia. Thromb Haemost. 2000;84:57682.

29. Pezzini A, Zotto E, Archetti S, Negrini R, Bani P, Albertini $A$, et al. CBS genotype in young adults with spontaneous cervical artery dissection plasma homocysteine concentration, C677T MTHFR genotype, and 844ins68bp and atherothrombotic stroke. Stroke. 2002;33:664-9.

30. Franco R, Reitsma PH. Genetic risk factors of venous thrombosis. Hum Genet. 2001;109:369-84. 